\title{
Classical conditioning of arterial pulse transit time and ECG-initiated transit time with the cold pressor as unconditioned stimulus
}

\author{
SELINA REDMAN and JOHN DUTCH \\ Victoria University, Wellington, New Zealand
}

\begin{abstract}
Arterial pulse transit time (PTT) and ECG-initiated transit time (ECG-TT) can be measured noninvasively in human subjects and reflect significant aspects of cardiovascular activity. In eight conditioning trials, with the cold pressor as the unconditioned stimulus, conditioned decreases in PTT and increases in ECG-TT were observed in later trials, concomitant with conditioned vasoconstriction. The results are discussed in terms of the potential contribution of PTT and ECG-TT in examining the effects of psychological processes on the cardiovascular system.
\end{abstract}

Classically conditioned cardiovascular responses have been widely investigated in animals (e.g., Powell \& Kazis, 1976; Yehle, Dauth, \& Schneiderman, 1967). However, with human subjects, although heart rate (e.g., Zeaman, Deane, \& Wegner, 1954) and vasomotor (Gottschalk, 1946; Shmavonian, 1959) conditioned responses (CRs) have been demonstrated, research has been hampered by the absence of suitable noninvasive measurement techniques to record additional aspects of cardiovascular function.

The purpose of this paper is to extend previous findings on classically conditioned cardiovascular responses in humans by investigating the conditioning of two responses, arterial pulse transit time (PTT) and the interval between the R-wave of the ECG and the arrival of the ensuing pulse at a peripheral site (ECG-TT). Although neither of these responses has previously been studied within a classical conditioning procedure, both reflect significant aspects of cardiovascular function and can be recorded noninvasively.

When measured between two sites on the same artery, PTT reflects arterial distensibility (Gow \& Taylor, 1968) and has been shown to covary inversely with changes in diastolic blood pressure (Bramwell, Downing, \& Hill, 1923; Geddes, Voelz, Babbs, Bourland, \& Tacker, 1981). ECG-TT may reflect changes in systolic blood pressure (Allen, Schneider,

This research was supported by Grant 200/13279 from the New Zealand Scientific Research Distribution Committee, by Grant 78/263 from the New Zealand University Grants Committee, and by V.U.W. IRC grants. This research was completed while the first author held a Medical Research Council of New Zealand postdoctoral fellowship. Requests for reprints should be sent to John Dutch, Department of Psychology, Victoria University of Wellington, Private Bag, Wellington, New Zealand.
Davidson, Winchester, \& Barr-Taylor, 1981) and is altered by variations in the duration of the preejection period which is sensitive to beta-adrenergic effects on the myocardium (Ahmed, Levinson, Schwartz, \& Ettinger, 1972).

\section{METHOD}

\section{Subjects}

Ten subjects, two females and eight males, volunteered to participate in the experiment.

\section{Equipment and Physiological Recording Procedures}

The presentation of stimuli and data collection was controlled by a Hewlett-Packard 9835B desktop computer.

The brachial pulse was transduced using a Narco Biosystems piezoelectric Korotkoff-sounds microphone, secured to the left arm with an elastic cuff. The digital pulse was recorded using a Beckman optical plethysmograph from the index finger of the left hand. The ECG signal was detected using Lead II (both arms and the left leg) with Beckman electrodes, and then preamplified using a Phillips PM 5140 LF generator. All signals were accoupled to a Beckman Offner dynograph, Type S, set on a low-frequency filter, $0.16 \mathrm{~Hz}$. The digital pulse signal was then amplified and filtered at LP $10 \mathrm{~Hz}$ to eliminate high-frequency noise. To overcome the inherent delay of the filter, the brachial pulse was also filtered at HP $0.01 \mathrm{~Hz}$, which minimally altered the signal.

The brachial and digital pulse signals were then fed into a dualchannel Gould advance storage oscilloscope (OS 4000). Each sweep of the oscilloscope was initiated by the R-wave of the BCG and the sweep speed was set at $0.05 \mathrm{~cm} / \mathrm{sec}$, so that both pulses were visible on the screen. The pulse signals were digitized by the oscilloscope and automatically output to the computer.

PTT was calculated from this data as the interval between the foot of the brachial pulse and the foot of the digital pulse, and ECG-TT was measured between the R-wave of the ECG (indicated by the extreme left-hand edge of the oscilloscope) and the foot of the brachial pulse. Prior to the experiment, the voltage level of each channel of the oscilloscope (i.e., the ground or zero level) was assessed and the foot of each pulse was defined as the first point on the pulse above this level. Pulse amplitude was calculated as the difference between this zero level and the pulse peak.

Heart rate was measured as interbeat interval (IBI) timed between successive R-waves of the ECG, using a Hewlett-Packard counter-timer (Model S328A), with millisecond accuracy. 


\section{Procedure}

A differential conditioning procedure was used with eight CS+ and eight CS - trials, randomly interspersed.

Each trial began with the collection of a 40-beat baseline immediately followed by the presentation of the CS + or CS- for 10 beats. On CS + trials, the experimenter, who was seated behind the subject, immersed the subject's hand in ice water for $\mathbf{4 0}$ beats. From a pilot study it was evident that recovery of the PTT and digital pulse height response to the cold pressor was not complete after $60 \mathrm{sec}$, and therefore an intertrial interval (ITI) of $4 \mathrm{~min}$ was used.

The CS+ and CS- stimuli were slides consisting of either a black triangle or a black circle superimposed on a green background. Half of the subjects received the triangle as CS+ and the circle as CS-; this was reversed for the remaining subjects. The stimuli were projected onto a screen directly in the subject's line of vision by a Kodak Carousel projector. The computer controlled both the slide-change mechanism and, since this was somewhat slow, operated a metal shutter over the lens of the projector, which permitted more precise timing of stimulus presentation. During the UCS, or immersion, the shutter obscured the projector lens. The use of a long ITI resulted in an extended session; in the pilot study, the subjects reported feeling extremely drowsy and bored, and marked tonic lengthening of IBI was apparent. In an attempt to attenuate the aversive effects of boredom, slides made from the work of a variety of well-known painters were presented during the ITI and baseline periods. Slide changes were randomly spaced with a mean display time of $2 \mathrm{~min}$, and the number of slides presented between trials ranged from one to five. This procedure had the additional advantage of both increasing the probability of subjects' watching the screen where the CS+/CS- stimuli were displayed and of aiding habituation of the initial response to the somewhat abrupt noise of the slide-change mechanism.

Upon arrival at the laboratory, the subjects were conducted to the experimental room, which was sound attenuated and kept at a temperature of $70^{\circ} \mathrm{F}$, and then were positioned supine upon a bed. The microphones and electrodes were attached, and the procedure briefly was explained. Headphones, through which music was played throughout the experiment, were then positioned. The room was darkened, and the subjects were left to relax for $5 \mathrm{~min}$ before any data collection began.

\section{RESULTS}

The median change from baseline in response to both CS+ and CS - presentation was calculated separately for each subject on each trial. The median rather than the mean was used because the mean is particularly open to distortions from extreme or erroneous readings. All IBI readings greater than $1.4 \mathrm{sec}$ or less than $0.45 \mathrm{sec}$ were discarded as erroneous.

Figure 1 illustrates both the percentage of subjects who showed a PTT decrease from baseline in response to the CS+ and those who made a greater PTT decrease to the CS+ than to the CS- for each trial. It is evident that the percentage of subjects responding in both these measures increased in the later trials, and it was therefore decided to evaluate Trials 1-4 separately from Trials 5-8 for all responses. The change from baseline in response to the CS+ was calculated separately for each subject for Trials 1-4 and Trials 5-8. The Wilcoxon matched-pairs test (two-tailed) was used to assess these data across subjects for each of the two trial blocks. A summary of

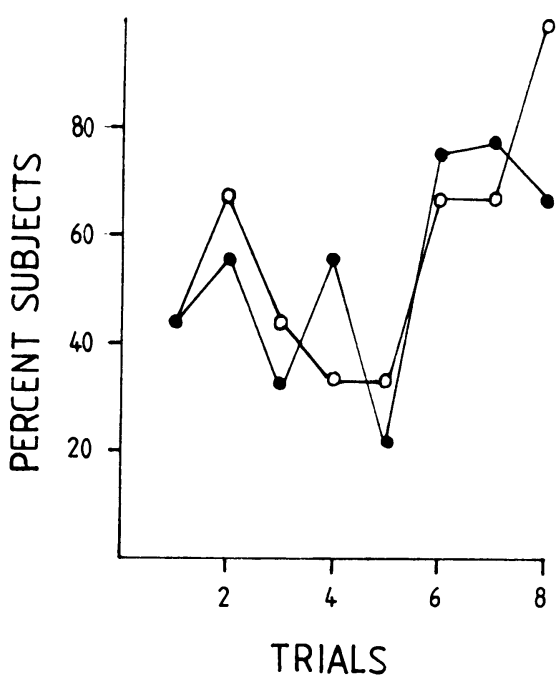

Figure 1. The percentage of subjects showing a decrease from baseline in response to the $\mathrm{CS}+$ (filled circles) and a greater decrease from baseline in response to the CS+ than to the CS(hollow circles) on each trial.

these changes is presented in Table 1. No comparisons were significant during the first four trials, but during Trials 5-8 a significant decrease in PTT $(p<.01)$, increase in ECG-TT $(p<.02)$, and decrease in pulse height $(\mathrm{p}<.01)$ occurred during CS+ presentation.

The difference between the change from baseline in response to the CS+ and that to the CS- was also calculated separately for each subject for Trials 1-4 and 5-8. The Wilcoxon matched-pairs test (twotailed) was again used to assess data across subjects, and a summary is presented in Table 1 . No comparisons were significant during the first four trials, but during Trials $5-8$ a greater decrease in PTT $(p<.05)$ and pulse height $(\mathrm{p}<.05)$ was observed in response to the CS+ than to the CS-.

Time-related changes or beat effects during the CS-UCS interval were also investigated. For each re-

Table 1

CS+ Compared With Baseline and With CS-, Averaged Across Subjects and Separately for Trials 1-4 and 5-8

\begin{tabular}{lccccc}
\hline & \multicolumn{2}{c}{ CS+ vs. Baseline } & & \multicolumn{2}{c}{ CS+ vs. CS- } \\
\cline { 2 - 3 } \cline { 5 - 6 } & $1-4$ & $5-8$ & & 1.4 & $5-8$ \\
\hline PTT & -.61 & $-5.19 \dagger$ & & .91 & $-5.30^{*}$ \\
ECG-TT & -.12 & $1.84^{*}$ & & .01 & 2.20 \\
AMP-Digital & -6.21 & $-7.15 \dagger$ & & -4.45 & $-5.73^{*}$ \\
IBI & -3.27 & -5.65 & & -17.04 & -10.23 \\
\hline
\end{tabular}

Note-All values are given as change scores from baseline, with PTT, ECG.TT, and IBI in milliseconds and amplitude of the digital pulse as percentage change from baseline. Negative values indicate a response to the CS+ of a greater decrease than either baseline or the response to the CS-. Significance levels are based on Wilcoxon matched pairs.

${ }^{*} p<.05 . \quad{ }^{* *} p<.02 . \quad t p<.01$. 


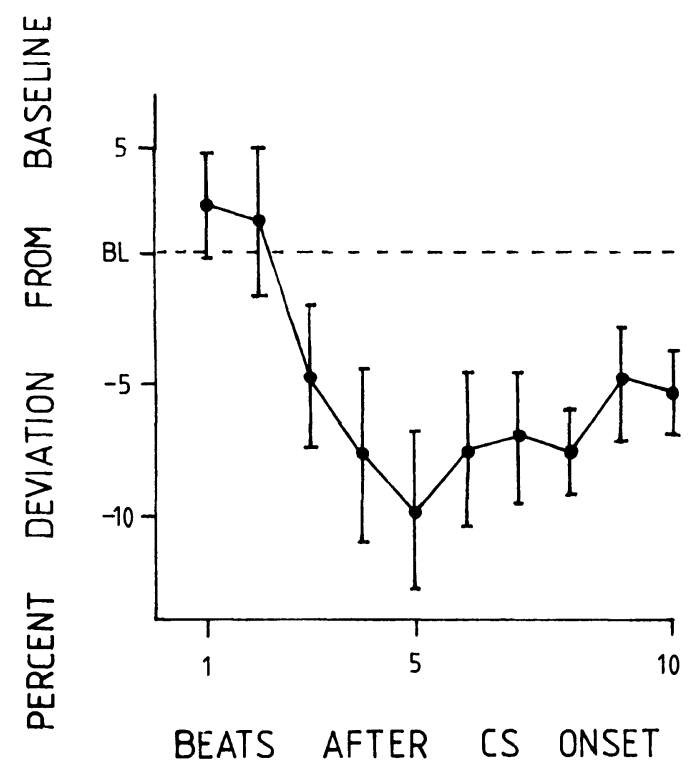

Figure 2. Beat-by-beat changes in the amplitude of the digital pulse (expreased as percentage change from baseline) during CS+ presentation, averaged across subjects during Trials 5-8.

sponse, the median change from baseline for each beat over the last four CS + trials was calculated and plotted separately for each subject. Consistent beat effects were observed only for pulse height. A summary of the mean change from baseline in pulse height for each beat averaged across subjects is presented in Figure 2, from which it is evident that the maximum change from baseline occurs between four and eight beats after CS onset.

\section{DISCUSSION}

Although there was no evidence of conditioned responding in early trials in any of the cardiovascular responses recorded, during the second four trials a CR was observed in several responses.

A conditioned decrease in PTT was found, both in comparison with baseline levels and with responding during the CS-. The decrease in PTT is likely to reflect increases in the rigidity of the artery wall (Gow \& Taylor, 1968) and in the level of diastolic blood pressure (Geddes et al., 1981).

When compared with baseline, a significant lengthening of ECG-TT was observed during CS+ presentation. Since PTT decreased during this period, the conditioned lengthening of ECG-TT is attributable to an extended pre-ejection period. This finding is consistent with evidence indicating that the pre-ejection period is lengthened by increases in vasoconstriction and diastolic blood pressure (Lewis, Leighton, Forester, \& Weissler, 1974; Soustre \& Rakotonirina, 1981), since decreases in pulse height and PTT were noted to occur in this experiment concurrently with the lengthening of ECG-TT. Differen- tial ECG-TT responding to the CS+ and CS - was not apparent. Although there was a clear trend towards a greater increase in transit time during the $\mathrm{CS}+$ in later trials, failure to find a significant difference was the result of a reduced $\mathbf{N}$ in this comparison. Due to a high proportion of movement artifact during CSpresentation, CS- data from two subjects could not be used.

In later trials, a conditioned decrease in pulse height was also evident. Since decreases in the height of the photoelectrically transduced pulse have been regarded as indicating vasoconstriction (e.g., Hogan, 1970), this experiment replicated the findings by Gottschalk (1946) and Shmavonian (1959) of a vasoconstrictive CR developed with the cold pressor as unconditioned stimulus.

Conditioned IBI responding was not observed. Although we are unaware of any reports of an IBI CR developed with the cold pressor, the failure to find conditioned responding is surprising given the consistent heart rate response reportedly elicited by the cold pressor (e.g., Obrist, Gaebelein, Teller, Langer, Grignolo, Light, \& McCubbin, 1978). Speculatively, the repeated application of such a noxious stimulus as the cold pressor (Wolf \& Hardy, 1941) may have raised baseline levels and thus confounded CR measures. Future research will attempt to elucidate this question.

In providing an initial demonstration of classically conditioned PTT and ECG-TT responses, this experiment indicates the sensitivity of these responses to psychological procedures. Given the previously demonstrated relationship of these responses to significant aspects of cardiovascular function and the noninvasive measurement procedure, PTT and ECGTT may provide important tools for research aimed at investigating the manner in which psychological processes affect the cardiovascular system. In particular, these responses may help to elucidate the role of classical conditioning in the development of essential hypertension through their relationship to such critical aspects of cardiovascular function as blood pressure, arterial elasticity, and betaadrenergic effects.

\section{REFERENCES}

Ahmed, S. S., \& Levinson, G. E., Schwartz, C. J., \& Ettinger, P. D. Systolic time intervals as measures of the contractile state of the left ventricular myocardium in man. Circulation, 1972, 46, 559-571.

Allen, R. A., Schneider, J. A., Davidson, D. M., Winchester, M. A., \& BARR-TAYLOR, C. The covariation of blood pressure and pulse transit time in hypertensive patients. Psychophysiology, 1981, 18, 301-306.

Bramwell, J. C., Downing, A. C., \& Hill, A. V. The effect of blood pressure on the extensibility of the human artery. Heart, 1923, 10, 289-300.

Geddes, L. A., Voelz, M. H., Babbs, C. F., Bourland, J. D., \& TACKER, W. A. Pulse transit time as an indicator of arterial blood pressure. Psychophysiology, 1981, 18, 71-74. 
Gotrschalk, L. A. A study of conditioned vasomotor responses in ten human subjects. Psychosomatic Medicine, 1946, 8, 16-27.

Gow, B. S., \& TAYLOR, M. G. Measurement of viscoelastic properties of arteries in the living dog. Circulation Research, 1968, 23, 111-122.

Hogan, D. H. Some physiological determinants to autonomic responsivity to sound. Journal of Speech and Hearing Research, 1970, 13, 130-146.

Lewis, R. P., Leighton, R. F., Forester, W. F., \& Weissler, A. M. Systolic time intervals. In A. M. Weissler (Ed.), Noninvasive cardiology. New York: Grune \& Stratton, 1974.

Obrist, P. A., Gaebelein, C. J., Teller, E. S., Langer, A. W., Grignolo, A., Light, K. C., \& McCubbin, J. A. The relationship among heart rate, carotid $\mathrm{dP} / \mathrm{dt}$ and blood pressure in humans as a function of the type of stress. Psychophysiology, 1978, 15, 102-115.

Powell, D. A., \& KAzis, E. Blood pressure and heart rate changes accompanying classical eyeblink conditioning in the rabbit (Oryctolagus cuniculus). Psychophysiology, 1976, 13, 441-447.
Shmavonian, B. M. Methodological study of vasomotor conditioning in human subjects. Journal of Comparative and Physiological Psychology, 1959, 52, 315-321.

Soustre, H., \& RAKotonirina, A. Electrophysiological and mechanical studies of frog heart adrenoceptor stimulation by epinine. Cardiovascular Research, 1981, 15, 700-710.

Wolf, S., \& HARDY, J. D. Studies on pain. Observations on pain due to local cooling and on factors involved in the "cold pressor" effect. Journal of Clinical investigation, 1941, 20, 521-533.

Yehle, A. L., Dauth, G. W., \& Schneiderman, N. Correlates of heart rate classical conditioning in curarized rabbits. Journal of Comparative and Physiological Psychology, 1967, 64, 98-104.

Zeaman, D., Deane, G., \& Wegner, N. Amplitude and latency characteristics of conditioned heart rate response. Jcurnal of Psychology, 1954, 38, 235-250.

(Manuscript received August 23, 1982; revision accepted for publication February 4, 1983.) 